\title{
Millimeter-Wave Periodic Surface Lattices for Mode Control in Vacuum Electronic Sources
}

\author{
A. J. MacLachlan ${ }^{1,2}$, A. R. Phipps ${ }^{1,2}$, C. W. Robertson ${ }^{1,2}$, I. V. Konoplev ${ }^{3}$, K. Ronald ${ }^{1,2}$, \\ A. W. Cross ${ }^{1,2}$ and A. D. R. Phelps ${ }^{1,2}$ \\ ${ }^{1}$ Department of Physics, SUPA, University of Strathclyde, Glasgow, G4 0NG, UK \\ ${ }^{2}$ Cockcroft Institute, Daresbury, Warrington, WA4 4AD, UK \\ ${ }^{3}$ JAI, Department of Physics, University of Oxford, Oxford, OX1 3RH, UK
}

\begin{abstract}
Periodic surface lattice (PSL) structures of planar geometry have been designed, constructed and measured. The motivation is to study the evolution of electromagnetic fields stimulated by PSLs to improve mode control in overmoded structures in high power electron beam driven $\mathrm{THz}$ vacuum electronic sources. The results of studies of the planar structures are presented. Prior to the experimental studies numerical simulations have been carried out and results are compared with experimental measurements. It is demonstrated that volume and surface fields can couple so as to form a cavity eigenmode, which has the potential to be selectively and efficiently excited.
\end{abstract}

Keywords-periodic surface lattice; surface wave; mode control; wave coupling; millimeter-wave sources

\section{INTRODUCTION}

To advance the performance parameters and extend the frequency range of vacuum electronic devices, electromagnetic structures that can enhance and control the mode selection process in the beam wave interaction region are being investigated. Various structures and materials such as diffractive surfaces [1], metamaterials [2], photonic structures [3], metadielectrics and periodic surface lattices [4] are being studied in different regimes of operation. The use of such diverse structures enables the wave dispersion to be engineered within its own discrete parameter space with often shared theoretical principles, and similar numerical modelling methods being used. This enables the structures to be designed and different advanced construction techniques such as photolithography, additive manufacturing, electrochemical deposition or high accuracy wire erosion to be used to manufacture the structures: with measurements of the electromagnetic properties of the structures carried out using similar experimental techniques such as Vector Network Analysis. By varying some of the parameters and especially the electromagnetic wavelength and structure size one can transition between the different regimes of operation.

Letizia et al. [5] have shown that photonic crystal structures can be used to improve the performance of $\mathrm{THz}$ vacuum electron devices. In their work a $0.650 \mathrm{THz}$ slow-wave BWO using photonic crystal structures was designed. In the case of mm-wave fast-wave devices the exploitation and control of electromagnetic fields inside and on the surface of periodic lattices has been shown by Ginzburg et al. [6] and Konoplev et al. [7] to be important for the realization of compact, highpower sources. Periodic surface lattices (PSLs) can be obtained by introducing periodic perturbations to a metal surface and

Part of this work has been sponsored by AFOSR under grant number FA8655-13-1-2132. The postgraduate research studentships of A. R. Phipps and A. J. MacLachlan were sponsored by the Engineering and Physical Sciences Research Council (EPSRC), UK. have been shown to facilitate coupling between volume and surface fields, resulting in the formation of a cavity eigenmode. In previous work, a dispersion relation describing coupling of volume and surface fields inside a 2D PSL of cylindrical topology was derived [8,9]. It was shown that, under certain conditions, the structure can support a slow-wave Cherenkov instability, demonstrating the potential of this coupled mode principle for high power, coherent sources

In the present "proof of principle" research planar structures with chessboard corrugations [10] have been investigated. The structure under investigation is made of a dielectric layer which is sandwiched between the PSL and a thin copper foil. This allows the trapping of a volume field between the PSL and the copper foil as in a Fabry-Perot cavity. This also has similarity with coaxial [11] and cylindrical [12] structures studied previously. Like in the previous studies the trapped volume field acts as a global oscillator (Fig. 1) which synchronizes the individual scatterers around which the localized surface fields are formed. This ensures synchronization of individual lattice elements, while the surface currents excited along the boundaries of each cell allow "cross-talk" between neighboring elements. Fig. 1 shows the schematic of the structure under investigation. The insert is the photograph of the section of PSL with the dark brown color shows dielectric while light brown corresponds to the metal surface. The rings indicating the passage of the surface currents and the narrow "bridges" are finite width "point" contacts between the metal squares. These point contacts have a large resistivity and do not allow "direct" current flows between the cells insuring coupling between individual cells only via EM fields. The volume field, if only a single mode is selected, works as an external force which synchronizes the surface fields i.e. the surface currents around each cell.

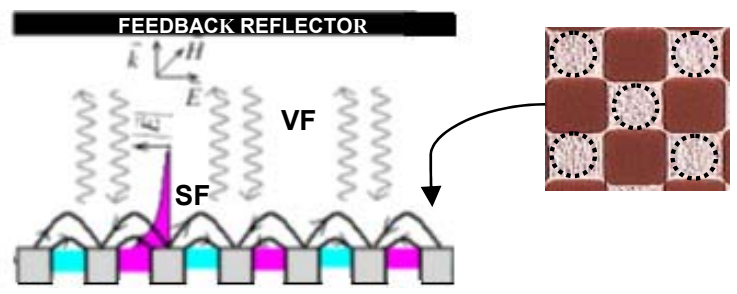

Fig.1. Schematic of the planar PSL structure

PSLs have been obtained through a process of chemical etching, using printed circuit board (PCB) with a $35 \mu \mathrm{m}$ copper coating and FR-4 dielectric (Fig.2a). 


\section{RESULTS}

A set of PSLs with period $d_{z}=1.50 \mathrm{~mm}, 1.62 \mathrm{~mm}, 1.74 \mathrm{~mm}$ and $1.94 \mathrm{~mm}$ have been etched onto PCB samples of various thicknesses $(0.4 \mathrm{~mm}, 0.8 \mathrm{~mm}$ and $1.6 \mathrm{~mm})$ both with and without the copper backing, in order to investigate the fundamental electromagnetic lattice properties. Scattering parameters of the PSLs are measured at various incident angles using a pair of Gband horns attached to high-frequency (140-220 GHz) heads and connected to a Vector Network Analyzer (VNA) as shown in Fig. 2(b).

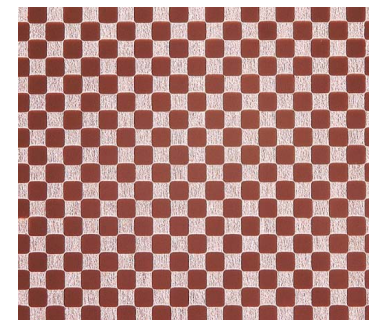

Fig.2(a) Copper planar PSL

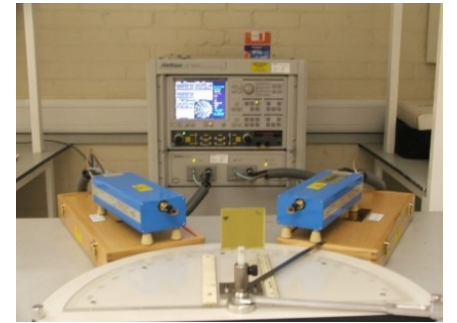

(b) Experimental set-up.
Increasing the dielectric thickness from $0.4 \mathrm{~mm}$ to $0.8 \mathrm{~mm}$, in the presence of the copper foil backing, results in much sharper resonances $(\sim-40 \mathrm{~dB})$. Although there is a slight frequency shift with period, the position of these resonances is limited within a narrow frequency range $(\sim 145-165 \mathrm{GHz})$ for all lattice periods. Here, the volume field inside the dielectric is coupling with the surface field at the periodic surface as desired, and resulting in eigenmode formation (Fig.3). Note that this phenomenon is not so clearly seen without the copper foil backing, which increases the cavity effect of the structure and synchronizes the perturbations.

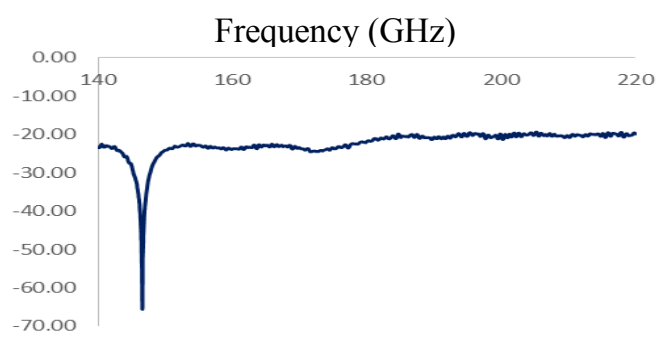

Fig.3 Reflected signal (dB) as a function of frequency showing coherent eigenmode formation due to coupling of surface and volume fields in PSL with period $1.94 \mathrm{~mm}$, dielectric thickness $0.8 \mathrm{~mm}$, and a $35 \mu \mathrm{m}$ copper backing.

The work in this paper has been extended by studying PSLs at higher frequencies in the $325-500 \mathrm{GHz}$ band. Numerical modelling has also been carried out to understand the coupling of volume and surface modes and the effect of varying dielectric and lattice parameters. Using CST Microwave Studio, a single unit cell is irradiated by plane waves over a range of angles and the scattering parameters are measured by a "Floquet" port. An alternative approach involves modelling a small section of the structure in order to provide a more realistic representation of the experiment by taking into account phenomena such as edge effects. All the PSLs considered in this work are scalable and the theory developed is applicable to a broad range of frequencies including $\mathrm{THz}$ radiation.

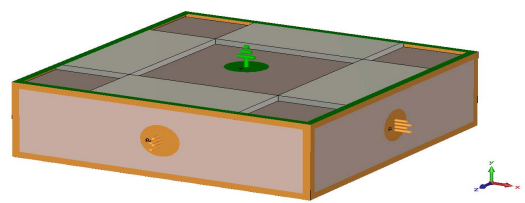

Fig. 4 Unit cell of the planar 2D PSL with periodic boundaries along $\mathrm{x}$ and $\mathrm{z}$, used to obtain a dispersion relation with the Eigenmode solver of CST Microwave Studio. $\mathrm{H}_{\mathrm{t}}=0$ at the corrugated surface and $\mathrm{E}_{\mathrm{t}}=0$ at the metal wall.

\section{CONCLUSIONS}

The coupling between volume and surface modes and coherent eigenmode formation in PSL structures has been successfully demonstrated. It has been shown that the parameters of these structures must be carefully chosen to facilitate the resonant coupling of modes. When the necessary conditions are met, these structures can provide an interaction region for novel, coherent sources of radiation in the microwave and millimeter-wave ranges. These sources are not, in principle, restricted in their frequency of operation and with suitable scaling could extend up to $\mathrm{THz}$ frequencies.

\section{ACKNOWLEDGMENT}

The authors thank David Barclay for his technical assistance.

\section{REFERENCES}

[1] H. L. Andrews, F. B. Taheri, I. V. Konoplev, et al "Reconstruction of the time profile of $20.35 \mathrm{GeV}$, subpicosecond long electron bunches by means of coherent Smith-Purcell radiation" Phys. Rev. Special Topics in Accelerators and Beams, vol. 17, art. no. 052802, May 2014.

[2] D. Shiffler, R. Seviour, E. Luchinskaya, et al, "Study of Split-Ring Resonators as a Metamaterial for High-Power Microwave Power Transmission and the Role of Defects", IEEE. Trans. on Plasma Science, vol. 41, pp. 1679-1685, June 2013.

[3] R. Letizia, M. Mineo and C Paoloni, "Photonic Crystal-Coupler for Sheet Beam THz Vacuum Electron Tubes", IEEE. Trans. Electron Devices, vol. 37, pp. 1227-1230, Sept. 2016.

[4] A. J. MacLachlan, A. R. Phipps, C. W. Robertson, et al., "Planar Periodic Surface Lattices for Use in Millimeter-Wave Sources", $39^{\text {th }}$ International Conference on Infrared, Millimeter, and Terahertz Waves, 978-1-4799-3877-3; 10.1109-IRMMW-THz.2014.6956011, Sept. 2014.

[5] R. Letizia, M. Mineo, and C. Paoloni, "Photonic Crystal-Structures for THz Vacuum Electron Devices”, IEEE. Trans. Electron Devices, vol. 62, pp. 178-183, Jan. 2015.

[6] N. S. Ginzburg, N. Y. Peskov, A. S. Sergeev, et al. , "Theory of freeelectron maser with two-dimensional feedback driven by an annular electron beam", J. Appl. Phys., vol. 92, pp. 1619-1629, Aug. 2002.

[7] I. V. Konoplev, A. W. Cross, A. D. R. Phelps, et al., "Experimental and theoretical studies of a coaxial free-electron maser based on twodimensional distributed feedback", Phys. Rev. E, vol. 76, art. no. 056406, Nov. 2007

[8] I. V. Konoplev, A. J. MacLachlan, C. W. Robertson, et al., “Cylindrical periodic surface lattice as a metadielectric: concept of a surface-field Cherenkov source of coherent radiation", Phys. Rev. A, vol. 84, art. no. 013826 , July 2011.

[9] I. V. Konoplev, A. J. MacLachlan, C. W. Robertson, et al., "Cylindrical, periodic surface lattice - Theory, dispersion analysis and experiment", Appl. Phys. Lett., vol. 101, art. no. 121111, Sept. 2012.

[10] A. J. MacLachlan, "Control, Manipulation and Exploitation of Electromagnetic Radiation using Complex Electrodynamic Structures", PhD Thesis, University of Strathclyde, Dec. 2015.

[11] I. V. Konoplev, P. McGrane, W. He, et al., "Experimental study of coaxial free-electron maser based on two-dimensional distributed feedback", Phys. Rev. Lett., vol. 96, art. no. 035002, Jan. 2006.

[12] I. V. Konoplev, A. R. Phipps, A. D. R. Phelps, et al., "Surface field excitation by an obliquely incident wave", Appl. Phys. Lett., vol. 102, art. no. 141106, April 2013. 